\title{
Chronologic Changes of Nitric Oxide Concentration in the Cochlear Lateral Wall and Its Role in Noise-Induced Permanent Threshold Shift
}

Yuh-Shyang Chen, MD; Fen-Yu Tseng, PhD; Kai-Nan Lin, MD; Ting-Hua Yang, MD; Shoei Yn Lin-Shiau, PhD; Chuan-Jen Hsu, PhD

Objective: The objective of this study was to investigate the chronologic changes of nitric oxide (NO) concentration in the cochlear lateral wall and to explore its possible role in permanent threshold shift (PTS) after intense noise exposure.

Materials and Methods: Seventeen guinea pigs were subjected to a single continuous exposure to broadband white noise at $105 \pm 2 \mathrm{~dB}$ sound pressure level (SPL) for 40 hours and were divided into four groups according to various postnoise recovery periods. Another 12 guinea pigs were not exposed to noise and served as controls. The hearing status of all animals was evaluated with auditory brainstem responses (ABR) evoked by condensation "click" sounds. ABR were recorded both prior to noise exposure and immediately before killing the animal. After death, $\mathrm{NO}$ concentration in the cochlear lateral wall was directly measured with an NO/ozone chemiluminescence technique.

Results: An approximately 1.7-fold increase in NO concentration was observed immediately postnoise exposure, which persisted for up to 28 days. The threshold of ABR elevation (mean, $30 \mathrm{~dB}$ SPL) peaked immediately after cessation of noise exposure and gradually resolved to a PTS (mean, 14.5 dB SPL) 56 days after noise exposure when NO concentration had returned to its prenoise exposure level.

Conclusion: Noise-induced threshold shift, which resolved to a mild PTS, can be partially attributed to NO elevation in the cochlear lateral wall. Our results revealed a nonlinear correlation between $\mathrm{ABR}$ recovery

From the Department of Otolaryngology (Y.-S.C., K.-N.L., T.-H.Y.), the Department of Internal Medicine (F.-Y.T.), and the Pharmacological Institute (s.Y.L.-S.), College of Medicine, National Taiwan University, Taipei, Taiwan.

Editor's Note: This Manuscript was accepted for publication December $12,2007$.

Send correspondence to Chuan-Jen Hsu, Department of Otolaryngology, National Taiwan University Hospital, No. 7, Chung-Shan South Road, Taipei, Taiwan, ROC. E-mail: cjhsu@ntu.edu.tw

DOI: 10.1097/MLG.0b013e3181651c24 and depletion of $\mathrm{NO}$, indicating that the mechanisms of NO changes in the cochlear lateral wall may be more complicated than previously conceived and that other pathophysiologic mechanisms may also play important roles in noise-induced PTS.

Key Words: Noise, nitric oxide, auditory brainstem response, permanent threshold shift, cochlear lateral wall.

Laryngoscope, 118:832-836, 2008

\section{INTRODUCTION}

Noise overstimulation can induce a threshold shift in both humans and animals. There are many possible pathophysiologic mechanisms that may contribute to noise-induced hearing loss (HL). ${ }^{1-7}$ The cochlear lateral wall is known to play an important role in maintaining the endocochlear potential, ion transport, and regulation of endolymph balance. Hirose and Liberman ${ }^{8}$ showed the pathologic changes of the cochlear lateral wall including microcirculation dysfunction, strial swelling, degeneration of intermediate and marginal cells, as well as endocochlear potential threshold shift after noise stimulation. They inferred that the noise-induced threshold shift was attributed, at least partially, to abnormal endocochlear potential resulting from damaged stria and spiral ligament of the cochlear lateral wall.

Nitric oxide (NO) has been directly detected in the stria vascularis and spiral ligament of the cochlear lateral wall as well as the outer hair cells, inner hair cells, supporting cells, and ganglion cells of guinea pig cochlea. ${ }^{9,10}$ $\mathrm{NO}$, a stable and nonreactive free radical, is known to regulate normal vascular tone and causes vasodilatation and inhibits platelet adhesion and local thrombosis, thereby increasing blood flow. On the other hand, NO can react with superoxide $\left(\mathrm{O}_{2}^{-}\right)$or other free radicals to produce peroxynitrite $\left(\mathrm{ONOO}^{-}\right)$, a powerful and toxic oxidant that can damage a wide range of biological molecules.

Increasing evidence has been presented to suggest that NO plays an important role in noise-induced cochlear 
damage and hearing dysfunction. The formation of NO under noise stimulation may be enzyme dependent through NO synthases (NOS) ${ }^{11-13}$ or enzyme independent through direct disproportion or reduction of nitrite to NO under acidic or ischemic conditions. ${ }^{14-16}$ Shi et al. ${ }^{12}$ found that more intense signals of inducible NOS fluorescence were observed in the stria vascularis as well as hair cells of the cochlea of mice and guinea pigs after exposure to a $110 \mathrm{~dB}$ sound pressure level (SPL) broadband noise of 3 hours per day for 3 consecutive days. Shi and Nuttall ${ }^{13}$ also found an elevation of NO concentration and reactive oxygen species in the stria vascularis associated with pathologic changes of marginal cells and blood vessel wall damage, triggering the apoptotic process of these cells in the stria vascularis. They inferred that increased production of both $\mathrm{NO}$ and reactive oxygen species after noise stress may lead to marginal cell pathology and the dysfunction of cochlear microcirculation by inducing blood vessel wall damage.

However, direct quantitative evidence of NO production in the cochlear lateral wall related to noise-induced $\mathrm{HL}$ is very rare. The association of NO concentration in the cochlear lateral wall and auditory brainstem response (ABR) threshold shift after noise exposure were discussed in our previous animal study. ${ }^{17}$ Subsequent to noise exposure (105 dB SPL, $10 \mathrm{~min}$ ), an immediate three-fold elevation of NO concentration in the cochlear lateral wall was observed along with a temporary threshold shift (TTS) (mean, $16.2 \mathrm{~dB}$ ), both of which returned to a prenoise level within 1 week. Despite years of investigation into the mechanisms of noise-induced HL, the differences between TTS and permanent threshold shift (PTS) remain unclear. In the present study, we intended to detect directly the chronologic changes of NO concentration in the cochlear lateral wall after a continuous exposure to an intermediate noise (broadband white noise, $105 \pm 2 \mathrm{~dB}$ SPL for $40 \mathrm{hr}$ ) and observe the consequential changes in auditory threshold so as to compare the differences between noise-induced TTS and PTS as well as understand the possible role of NO in noise-induced PTS.

\section{MATERIALS AND METHODS}

\section{Animals}

A total of 29 healthy, adult male Hartley albino guinea pigs (250-300 g, 5-6 wk old) with normal thresholds of ABR (range, 20-30 dB SPL) for all ears were included in this study. Seventeen guinea pigs were subjected to a single continuous exposure to broadband white noise $(125-15 \mathrm{KHz})$ at approximately $105 \pm 2$ $\mathrm{dB}$ SPL for a period of 40 hours to induce a permanent auditory threshold shift (PTS). These test animals were then divided into four groups ( $\mathrm{N}-1$ to $\mathrm{N}-4)$ corresponding to various postnoise recovery periods $(0,7,28$, and $56 \mathrm{~d}$, respectively), as described in Table I.

During noise exposure, the animals were kept in a cage inside a soundproof room. White noise was produced by an audiometer (Grason-Stadler Inc., model GSI-10, Milford, NH) and presented by a set of loudspeakers located on either side of the cage in which the animals were kept. The SPL of the noise was measured with a sound level meter (Rion, model NA-24, Tokyo, Japan). The remaining 12 guinea pigs were not exposed to noise and served as controls. To rule out the effects of post-trauma survival time on ABR threshold and NO concentration, the controls were divided into three groups (C-1, C-2, and C-3) that were killed after an interval of 0,28 , and 56 days, respectively.

\section{Auditory Brainstem Response Recording}

An evoked potential system (Nicolet, Spirit, Madison, WI) was used to measure the ABR thresholds. The hearing status of all animals was evaluated with condensation "click" sounds (duration $100 \mu \mathrm{s}$, stimulation rate $57.7 / \mathrm{s}$, frequency $0-10,151 \mathrm{~Hz}$ ) evoked ABR. Animals were anesthetized with an intraperitoneal injection of pentobarbital $(35 \mathrm{mg} / \mathrm{kg}$ ). The procedure for $\mathrm{ABR}$ measurement was as previously described. ${ }^{6}$ For all noise-exposed groups, the ABR thresholds were measured prior to noise exposure and then measured again immediately prior to animal death. For the nonexposed control groups, the ABR thresholds were measured immediately prior to killing the animal after a survival time of 0,28 , and 56 days, respectively. Changes in the auditory function of the animals from the experimental groups were assessed by determining the difference in ABR thresholds for wave $\mathrm{V}$ between pre-exposure and predeath guinea pigs.

TABLE I.

Auditory Brainstem Response (ABR) Thresholds (mean \pm SD dB SPL) and Concentrations of Nitric Oxide (mean $\pm \mathrm{SD} \mathrm{nmol} / \mathrm{g}$ ) in Cochlear Lateral Walls of Various Noise-Exposure and Control Groups.

\begin{tabular}{lcccc}
\hline Group (Ears) & Survival (days) & NO/Pr $(\mathrm{nmol} / \mathrm{g})$ & $\begin{array}{c}\text { ABR Threshold } \\
\text { (Pre-Exposure) }\end{array}$ & ABR Threshold (Final) \\
\hline N-1 $(\mathrm{n}=10)$ & 0 & $17.44 \pm 1.30^{*}$ & $25.00 \pm 4.59$ & $55.00 \pm 6.58 \dagger$ \\
$\mathrm{N}-2(\mathrm{n}=6)$ & 7 & $18.16 \pm 2.33^{*}$ & $24.00 \pm 1.20$ & $48.16 \pm 6.65 \dagger$ \\
$\mathrm{N}-3(\mathrm{n}=8)$ & 28 & $18.36 \pm 2.29^{*}$ & $24.62 \pm 1.76$ & $43.38 \pm 6.78 \dagger$ \\
$\mathrm{N}-4(\mathrm{n}=10)$ & 56 & $10.82 \pm 3.06$ & $25.00 \pm 4.38$ & $39.00 \pm 5.66 \dagger$ \\
C-1 $(\mathrm{n}=8)$ & 0 & $10.32 \pm 0.74 \ddagger$ & $25.00 \pm 3.94 \S$ & $25.00 \pm 3.94$ \\
C-2 $(\mathrm{n}=8)$ & 28 & $10.63 \pm 1.52 \ddagger$ & $23.29 \pm 3.85 \S$ & $24.20 \pm 3.50$ \\
C-3 $(\mathrm{n}=8)$ & 56 & $10.59 \pm 0.85 \ddagger$ & $24.50 \pm 3.36 \S$ & $23.8 \pm 3.80$ \\
\hline \hline
\end{tabular}

${ }^{*} P<.005$, as compared with control group $\mathrm{C}-1$ ( $t$ test).

$+P<.005$, as compared with pre-exposure ABR level (paired $t$ test).

$\ddagger P>0.05$, as compared NO concentration among control groups (C-1, C-2, C-3) (analysis of variance, $P=.789$ ).

$\S P>.05$, as compared ABR threshold among control groups (C-1, C-2, C-3) (analysis of variance, $P=.5323$ ).

$\mathrm{NO}=$ nitric oxide; $\mathrm{Pr}=$ protein; $\mathrm{N}=$ noise-induced permanent threshold shift group; $\mathrm{C}=$ control group, without noise exposure. 


\section{Tissue Collection}

Immediately subsequent to the final measurement of ABR, the animals were decapitated. After decapitation, the temporal bones were quickly removed, opened to expose the otic capsule, and then stored at $-70^{\circ} \mathrm{C}$ until microdissection was performed. With microdissection techniques, the lateral wall of the individual cochlea, including the stria vascularis and spiral ligaments, was collected in $0.6 \mathrm{~mL}$ dilution buffer (mannitol $310 \mathrm{mmol} / \mathrm{L}$, HEPES $25 \mathrm{mmol} / \mathrm{L}$ ) in a $2 \mathrm{~mL}$ microcentrifuge tube, homogenized by ultrasonication (Sonics and Materials, Inc., VC-600, Danbury, $\mathrm{CT}$ ), and stored at $-10^{\circ} \mathrm{C}$ until the $\mathrm{NO}$ assay was performed. The care of the animals used in this study was approved by the Institutional Laboratory Animal Care Committee of the National Taiwan University, College of Medicine (\# 20030064).

\section{Measurement of NO}

To avoid incomplete denaturation of total protein, $1.17 \mathrm{~N}$ perchloric acid $\left(\mathrm{HClO}_{4}\right)$ was added to the homogenized tissue overnight (12-16 hr). Samples were subsequently centrifuged at $14,000 \mathrm{~g}$ for 20 minutes. The supernatants of these samples were collected and assayed by the NO/ozone chemiluminescence method (NO Analyzer 280A, Sievers Research, Inc., Boulder, CO). This quantitative NO assay method has been described previously. ${ }^{17}$ We measured the oxidation products $\left(\mathrm{NO}_{2}^{-}\right.$and $\left.\mathrm{NO}_{3}^{-}\right)$of $\mathrm{NO}$ using a reaction vessel containing a reducing system $(0.1 \mathrm{~mol} / \mathrm{L}$ vanadium chloride, SigmaAldrich, Munich, Germany). NO was then detected through its reaction with ozone, which leads to the emission of red light (NO + $\mathrm{O}_{3} \rightarrow \mathrm{NO}_{2} *+\mathrm{O}_{2} ; \mathrm{NO}_{2} * \rightarrow \mathrm{NO}_{2}+h v$ ). The linearity of the standard curves was confirmed with $5,2.5,1.25,0.625,0.31$, and $0.15 \mu \mathrm{mol} / \mathrm{L}$ NO samples, which were prepared using fresh solutions of $\mathrm{NaNO}_{2}$ $(10 \mu \mathrm{L})$ in distilled water.

\section{Protein Determination}

The protein content was determined by the bicinchoninic acid (BCA) method, as described previously, ${ }^{17}$ using bovine serum albumin (BSA) as the test standard. The assay protocol used a total assay volume of $120 \mu \mathrm{L}$ ( $10 \mu \mathrm{L}$ of sample or standard, $10 \mu \mathrm{L}$ of $0.1 \mathrm{~N} \mathrm{NaOH}$, and $100 \mu \mathrm{L} \mathrm{BCA}$ ) to determine protein content. Subsequent to the acquired results, the linearity of the standard curve was confirmed. The individual homogenate $(10 \mu \mathrm{L})$ of the lateral wall of the individual guinea-pig cochleae was incubated for 30 minutes at $37.0^{\circ} \mathrm{C} \pm 0.5^{\circ} \mathrm{C}$ in a $\mathrm{BCA}$ working reagent, after which the optical density of the solution was determined at 570 $\mathrm{nm}$ by an enzyme-linked immunosorbent assay reader (Dynatech, MR-7000, Ashford, Middlesex, UK). Total protein ( $\mu \mathrm{g})$ was determined by comparison with a standard BSA curve.

\section{Statistical Analysis}

Statistical comparisons between the study groups were performed using the Student $t$ test or the analysis of variance (ANOVA) test. The time trend of the measurements was presented graphically and analyzed by differences between the groups. Statistical significance was defined as $P<.05$. Analysis was performed using SPSS 8.0 for Windows (SPSS, Inc., Chicago, IL).

\section{RESULTS}

\section{Hearing Thresholds of ABR}

The mean ABR thresholds of the noise-exposed groups and the control groups are summarized in Table I, and the means of the pre-exposure and final ABR thresholds of the noise-exposed groups are depicted in Figure 1. Hearing thresholds measured with ABR were all elevated in the noise-exposed groups. The difference between the pre-exposure and final $A B R$ thresholds of the same indi-

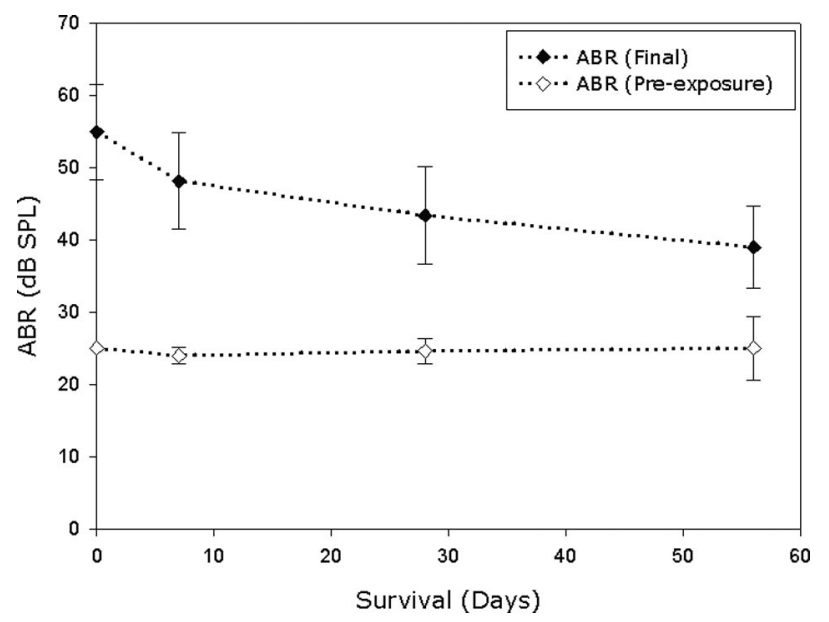

Fig. 1. Trends of means of pre-exposure and final auditory brainstem response thresholds ( $\mathrm{dB}$ sound pressure level) among the noise-exposed groups with various survival times (days).

vidual's ears proved to be statistically significant for all noise-exposed groups $(P<.005$; paired $t$ test). After noise exposure, the average threshold shift ranged from $30.00 \pm$ 6.60 to $14.50 \pm 3.54 \mathrm{~dB}$ SPL in groups with different survival periods. The hearing threshold showed partial recovery after termination of noise exposure but did not return to the pre-exposure levels even at 56 days postexposure. A $14.50 \mathrm{~dB}$ (mean) PTS occurred subsequent to the animal's continuous exposure to broadband white noise at $105 \pm 2 \mathrm{~dB}$ SPL for 40 hours. The means of the final ABR thresholds for the three control groups revealed no statistically significant difference (ANOVA, $P=.5323$ ), suggesting no post-trauma survival time effects on $\mathrm{ABR}$ threshold over the 56-day period.

\section{NO Concentration}

Changes in NO concentration at various survival times after noise exposure are shown in Table I, and the trend of chronologic changes to NO concentration is depicted in Figure 2. Immediately subsequent to the 40 hour noise exposure, $\mathrm{NO}$ concentration rose and remained high for at least 28 days postnoise exposure during the follow-up period. At 56 days postnoise exposure, NO concentration declined and demonstrated no significant difference with the prenoise exposure level. Likewise, the corresponding NO concentrations of the three control groups revealed no significant differences between them (ANOVA, $P=.789$ ), suggesting that there was no posttrauma survival time effects on the NO concentration of the cochlear later wall over the 56-day period.

Although the ABR threshold shift and NO elevation recovered gradually during the follow-up period, a final PTS $(14.50 \pm 3.54 \mathrm{~dB})$ remained, whereas the NO concentration returned to normal control levels by 56 days after noise exposure. This revealed a nonlinear correlation between ABR recovery and depletion of NO in the cochlear lateral wall in noise-induced PTS.

\section{DISCUSSION}

The present results showed three important findings in the long-term observation of NO production in the 


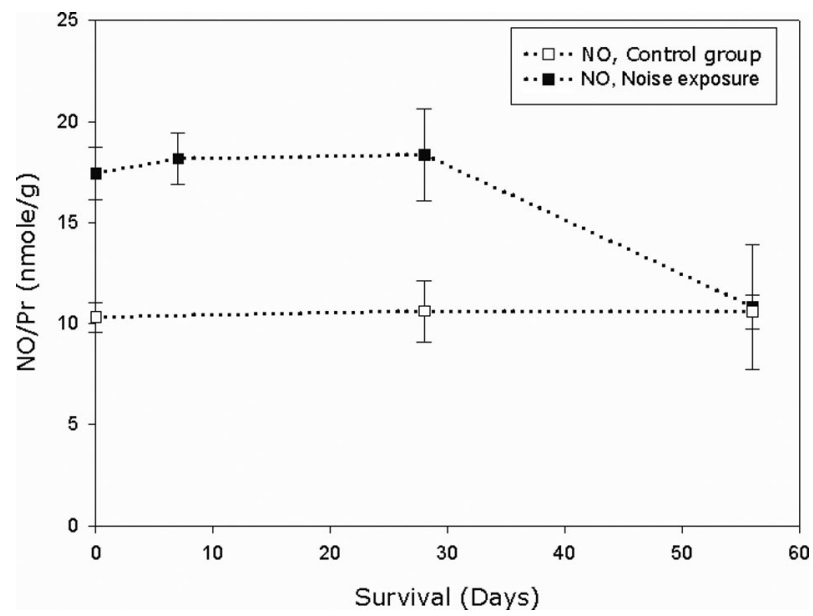

Fig. 2. Chronologic changes of nitric oxide concentration of the control and postnoise exposure groups during 56-day observation period.

cochlear lateral wall after a continuous noise exposure of intermediate intensity: 1) the maximal auditory threshold shift occurs immediately after cessation of noise stimulation, and a gradual recovery to a mild PTS is observed by 56 days postexposure; 2) after cessation of noise stimulation, NO production is maintained at a maximal or near maximal level for at least 28 days before finally returning to pre-exposure levels by 56 days postexposure; and 3) there is inconsistency between the chronologic changes of $\mathrm{NO}$ concentration in the cochlear lateral wall and ABR threshold shift after noise exposure.

In the present study, the experimental guinea pigs exhibited a significant threshold shift that resolved to a PTS of $14.50 \pm 3.54 \mathrm{~dB}$ by 56 days after noise exposure. We offer new evidence confirming that elevated NO concentration in the cochlear lateral wall is associated with auditory threshold shift after noise exposure. A maximal auditory threshold shift and a high NO concentration are both observed immediately after continuous noise exposure of 40 hours. It appears that an above-baseline NO level in the cochlear lateral wall after long-term noise overexposure is involved in the noise-induced PTS, especially at the initial postexposure period.

Initial NO concentrations in the cochlear lateral wall appear the same in the present results for PTS as did our previous results for noise-induced TTS. ${ }^{17}$ However, NO overproduction appears to persist longer in this study. NO levels remained elevated for an unexpected 28 days and only returned to a normal level after 56 days. At the same time, the ABR threshold shift showed only partial recovery, resulting in a mild PTS. We would posit several possible explanations for the nonlinear correlation between $\mathrm{ABR}$ recovery and depletion of $\mathrm{NO}$ in the cochlear lateral wall. First, the pathologic overproduction of NO and subsequent peroxynitrite formation is the important factor responsible for injury of the cochlear microcirculation and auditory dysfunction. The formation of peroxynitrite is extremely rapid, and the half-life is short, and therefore the long duration of NO production may accelerate and keep the peroxynitrite at toxic levels for a longer time, inducing a more severe damage to the cochlear tissue immediately after termination of noise exposure, as shown by the present study. However, under long-term noise exposure, the biochemical materials are consumed, and if there is not enough superoxide anions $\mathrm{O}_{2}^{-}$or other free radicals, the production of peroxinitrite cannot continuously accumulate to toxic levels to initiate new dysfunctional cells.

Second, a concentration of NO higher than the prenoise exposure level served a protective role in inducing recovery of the cochlear vascular homeostasis and partial recovery of the auditory function as well as damaged cells. Therefore, in the present study, the partial recovery of ABR threshold progressed as long as NO concentration remained higher than normal or higher than the preexposure level from the termination of noise exposure to 28 days after.

Third, the end result of auditory function as well as cochlear damage is not the result of continuous initiation of new dysfunction cells but the degradation of some existing dysfunctional cells during or shortly after exposure of intense noise and the recovery of other existing dysfunctional cells during the recovery period. The end ABR threshold of noise-induced HL may depend on the duration of peroxynitrite toxic levels during noise exposure, which may not parallel the NO level in the cochlear lateral wall. In other words, the duration and toxic levels of peroxynitrite may play a role in determining whether the noise-induced threshold shift will be temporary or permanent.

In addition, the mechanisms of noise-induced PTS cannot be simply explained as an NO-mediated process. It is well known that overstimulation of the cochlea by intense noise is a metabolic as well as a mechanical process. ${ }^{1,3,4}$ The relative importance of the two mechanisms in the shifting of the noise-induced hearing threshold may depend on the length and intensity of noise exposure. NO overproduction in the cochlear lateral wall only partially contributes to the ABR threshold shift, and other pathophysiologic mechanisms may play more important roles in noise-induced PTS. Moreover, noise-induced PTS is attributed not only to the damage to the spiral ligament and stria vascularis in the cochlear lateral wall but also to damage of cochlear hair cells. Chronologic changes in NO concentration were investigated only in the cochlear lateral wall in our present study.

Because NO can act as a double-edged sword, ${ }^{13,18-21}$ it may play different roles in generating overall noiseinduced cochlear dysfunction and destruction. The role of peroxynitrite overproduction, as well as the relationship between NO and peroxynitrite overproduction in noiseinduced threshold shift, needs further study. More detailed knowledge related to the prevalence and the time course of different effects of NO and peroxynitrite is of crucial importance in exploring potential therapeutic strategies for noise-induced HL. The critical timing of applying antioxidants as otoprotectors must be investigated because they may not only inhibit excess NO production but also repress cochlear microcirculation, resulting in irreversibly damaged cells. Therefore, effective hearing protective strategies may require adjustments at several time points 
during the period of NO production. Further studies are warranted to elucidate these issues.

In conclusion, the present results reveal a nonlinear correlation between $\mathrm{ABR}$ recovery and depletion of $\mathrm{NO}$ in the recovery stage. In noise-induced PTS, the mechanisms of NO changes in the cochlear lateral wall may be more complicated than previously thought, and other pathophysiologic mechanisms may also play important roles. Hearing protective strategies with antioxidants for noiseinduced HL should be further investigated.

\section{BIBLIOGRAPHY}

1. Spoendlin H. Organisation of the auditory receptor. Rev Laryngol Otol Rhinol (Bord) 1976;97(Suppl):453-462.

2. Canlon B, Schacht J. Acoustic stimulation alters deoxyglucose uptake in the mouse cochlea and inferior colliculus. Hear Res 1983;10:217-226.

3. Lim DJ. Effects of noise and ototoxic drugs at the cellular level in the cochlea: a review. Am J Otolaryngol 1986;7: 73-99.

4. Gao WY, Ding DL, Zheng XY, Ruan FM, Liu YJ. A comparison of changes in the stereocilia between temporary and permanent hearing losses in acoustic trauma. Hear Res. 1992;62:27-41.

5. Patuzzi R. Exponential onset and recovery of temporary threshold shift after loud sound: evidence for long-term inactivation of mechano-electric transduction channels. Hear Res 1998;74:217-220.

6. Hsu CJ, Shau WY, Chen YS, Liu TC, Lin-Shiau SY. Activities of $\mathrm{Na}^{+}, \mathrm{K}^{+}$-ATPase and $\mathrm{Ca}^{2+}$-ATPase in cochlear lateral wall after acoustic trauma. Hear Res 2000;142: 203-211.

7. Goldwin B, Khan MJ, Shivapuja B, Seidman MD, Quirk WS. Sarthran preserves cochlear microcirculation and reduces temporary threshold shifts after noise exposure. Otolaryngol Head Neck Surg 1998;118:576-583.
8. Hirose K, Liberman MC. Lateral wall histopathology and endocochlear potential in the noise-damaged mouse cochlea. J Assoc Res Otol 2003;4:339-352.

9. Shi X, Ren T, Nuttall AL. Nitric oxide distribution and production in guinea pig cochlea. Hear Res 2001;153:23-31.

10. Takumida M, Anniko M. Detection of nitric oxide in the guinea pig inner ear, using a combination of adehyde fixative and 4,5-diaminofluorescein diacetate. Acta Otolaryngol 2001;121:460-464.

11. Shi X, Ren T, Nuttall AL. The electrochemical and fluorescence detection of nitric oxide in the cochlea and its increase following loud sound. Hear Res 2002;164:49-58.

12. Shi X, Dai C, Nuttall AL. Altered expression of inducible nitric oxide synthase (iNOS) in the cochlea. Hear Res 2003; $177: 43-52$

13. Shi X, Nuttall AL. Upregulated iNOS and oxidative damage to the cochlear stria vascularis due to noise stress. Brain Res 2003;967:1-10.

14. Weitzberg E, Lundberg JO. Nonenzymatic nitric oxide production in humans. Nitric Oxide 1998;2:1-7.

15. Zweier JL, Samouilov A, Kuppusamy P. Non-enzymatic nitric oxide synthesis in biological system. Biochim Biophys Acta 1999;1411:250-262.

16. Butler AR, Ridd JH. Formation of nitric oxide from nitrous acid in ischemic tissue and skin. Nitric Oxide 2004;10: 20-24.

17. Chen YS, Tseng FY, Liu TC, Lin-Shiau SY, Hsu CJ. Involvement of nitric oxide generation in noise-induced temporary threshold shift in guinea pigs. Hear Res 2005;203:94-100.

18. Dimmeler S, Zeiher AM. Nitric oxide and apoptosis: another paradigm for the double-edged role of nitric oxide. Nitric Oxide 1997;1:275-281.

19. Nagura M, Iwasaki S, Mizuta K, Mineta H, Umemura K, Hoshino T. Role of nitric oxide in focal microcirculation disorder of guinea pig cochlea. Hear Res 2001;153:7-13.

20. Ruan RS. Possible roles of nitric oxide in the physiology and pathophysiology of the mammalian cochlea. Ann N Y Acad Sci 2002;962:260-274.

21. Yamashita D, Jiang HY, Schacht J, Miller JM. Delayed production of free radicals following noise exposure. Brain Res 2004;1019:201-209. 